\title{
A study of the reactions of molecular hydrogen with small gold clusters
}

\author{
Sergey A. Varganov, Ryan M. Olson, and Mark S. Gordon \\ Department of Chemistry, Iowa State University, Ames, Iowa 50011 \\ Greg Mills and Horia Metiu \\ Department of Chemistry and Biochemistry, University of California Santa Barbara, Santa Barbara, \\ California 93106
}

(Received 30 October 2003; accepted 17 December 2003)

\begin{abstract}
This work presents a study of reactions between neutral and negatively charged $\mathrm{Au}_{n}$ clusters $(n$ $=2,3$ ) and molecular hydrogen. The binding energies of the first and second hydrogen molecule to the gold clusters were determined using density functional theory (DFT), second order perturbation theory (MP2) and coupled cluster $(\operatorname{CCSD}(\mathrm{T}))$ methods. It is found that molecular hydrogen easily binds to neutral $\mathrm{Au}_{2}$ and $\mathrm{Au}_{3}$ clusters with binding energies of $0.55 \mathrm{eV}$ and $0.71 \mathrm{eV}$, respectively. The barriers to $\mathrm{H}_{2}$ dissociation on these clusters with respect to $\mathrm{Au}_{n} \mathrm{H}_{2}$ complexes are $1.10 \mathrm{eV}$ and $0.59 \mathrm{eV}$ for $n=2$ and 3. Although negatively charged $\mathrm{Au}_{n}^{-}$clusters do not bind molecular hydrogen, $\mathrm{H}_{2}$ dissociation can occur with energy barriers of $0.93 \mathrm{eV}$ for $\mathrm{Au}_{2}^{-}$and $1.39 \mathrm{eV}$ for $\mathrm{Au}_{3}^{-}$. The energies of the $\mathrm{Au}_{2} \mathrm{H}_{2}^{-}$and $\mathrm{Au}_{3} \mathrm{H}_{2}^{-}$complexes with dissociated hydrogen molecules are lower than the energies of $\mathrm{Au}_{2}^{-}+\mathrm{H}_{2}$ and $\mathrm{Au}_{3}^{-}+\mathrm{H}_{2}$ by $0.49 \mathrm{eV}$ and $0.96 \mathrm{eV}$, respectively. There is satisfactory agreement between the DFT and $\operatorname{CCSD}(\mathrm{T})$ results for binding energies, but the agreement is not as good for barrier heights. (C) 2004 American Institute of Physics. [DOI: 10.1063/1.1647118]
\end{abstract}

\section{INTRODUCTION}

This paper is concerned with the adsorption and the dissociation of $\mathrm{H}_{2}$ on very small, neutral and negatively charged $\mathrm{Au}_{n}$ clusters $(n=2,3)$. Its main purpose is to study hydrogen chemistry on these clusters and to test the accuracy of the density functional method by comparing its results to those obtained by using the coupled cluster method $\operatorname{CCSD}(\mathrm{T}){ }^{1}$

The interest in hydrogen chemistry on gold clusters has been stimulated by the recent discovery that supported gold clusters catalyze the hydrogenation of ethylene, 1,3butadiene, 1-butene, ${ }^{2}$ acrolein, ${ }^{3}$ and the partial hydrogenation of acetylene to ethylene. ${ }^{4}$

Even more interesting is the partial oxidation of propylene to propylene oxide, by a mixture of hydrogen and oxygen, which is catalyzed by small Au clusters supported on $\mathrm{TiO}_{2} .{ }^{5}$ It is rather unusual that an oxidation reaction requires the presence of hydrogen, and the reason for this is not yet understood. About $30 \%$ of the hydrogen is converted to water, which is a waste of an expensive feedstock. Therefore, work that furthers our understanding of the role of hydrogen in this process may have practical consequences. Other reactions catalyzed by small gold clusters are described in several excellent review articles.

Most electronic structure calculations in the fields of catalysis or surface science are performed by using density functional theory (DFT). This theory, which has had notable successes in explaining a variety of observations, ${ }^{6}$ offers no $a$ priori criterion for assessing the magnitude of the errors. These can be established only by comparison with experiments and/or higher level calculations. It is therefore prudent to test the accuracy of DFT, for each new system, before embarking on extensive predictive calculations on large systems of practical interest. This is especially important since we have recently illustrated that DFT calculations do not account, qualitatively or quantitatively, for the adsorption of molecular oxygen on small gold clusters. ${ }^{7}$

Calculations and experiments on small gas-phase clusters play a central role in such comparisons. Negatively charged clusters are of interest because experiments on them are more readily available than on neutral clusters. Furthermore, they may play a role in catalysis by supported clusters, since the Au clusters tend to bind on oxygen vacancies ${ }^{8}$ that are electron rich $^{9}$ and are chemically active. ${ }^{10} \mathrm{~A}$ very interesting example of the activation of a small gold cluster by contact with an oxygen vacancy on $\mathrm{MgO}$ is provided by the beautiful work of Sanchez et al. ${ }^{11}$

Finally, note that most industrial catalysts use large clusters, containing thousands of $\mathrm{Au}$ atoms. It is not clear whether such large clusters are necessary, and small clusters may present an interesting alternative, especially since their properties change markedly when the number of atoms is changed or when they are electrically charged or alloyed. Thus, they offer more opportunities for performance optimization than very large clusters. Unfortunately, their use has been prevented so far by their propensity to coarsen and future uses of small clusters will have to solve this problem.

\section{COMPUTATIONAL METHODS}

The geometries determined using Hartree-Fock (HF) calculations were used as the initial structures for DFT and $M P 2^{12}$ geometry optimizations. If, during HF geometry optimization $\mathrm{H}_{2}$ did not bind to a Au cluster the optimization process was repeated with DFT and MP2 using a reasonable initial guess for the geometry. To ensure that the optimized geometries correspond to minima or transition states on their respective potential energy surfaces, the Hessian (matrix of 
energy second derivatives) was calculated and diagonalized. A Hessian with $n$ negative eigenvalues corresponds to an $n$th order saddle point. Therefore, a zeroth order saddle point is a minimum on the potential energy surface, and a first order saddle point is a transition state. Since even relatively simple potential energy surfaces can have several minima and transition states, the intrinsic reaction path (IRC) method, ${ }^{13}$ using the second order Gonzalez-Schlegel algorithm ${ }^{14}$ was used to connect the transition states with minima. This technique guarantees that one has found the correct transition state connecting two known minima. The optimized MP2 geometries were then used for single point $\operatorname{CCSD}(\mathrm{T})$ energy calculations. The energy gradients for geometry optimizations were evaluated analytically for all HF, DFT, and closed shell MP2 calculations. For open-shell MP2 geometry optimizations the gradients were calculated numerically.

To avoid spin contamination in the wave function, restricted open shell methods were used for all open shell calculations. For open shell second order perturbation theory the Z-averaged perturbation theory $\left(\mathrm{ZAPT}^{15}\right)$ version of restricted MP2 was used. The ZAPT and the restricted $\operatorname{RCCSD}(\mathrm{T})^{16}$ method are based on a restricted open shell HF wave function that is free of spin contamination. In the MP2 and $\operatorname{CCSD}(\mathrm{T})$ calculations the $1 s$ orbitals of oxygen and $5 s$ and $5 p$ core orbitals of gold atoms were not included in the correlation part of the calculation.

The restricted DFT method with the B3LYP ${ }^{17}$ functional was used to compare with the results of plane-wave-based DFT calculations and with the MP2 and $\operatorname{CCSD}(\mathrm{T})$ calculations described above. The B3LYP functional consists of five functionals, namely Becke + Slater + HF exchange and LYP +VWN5 correlation.

In all calculations the effective core potential (ECP) with scalar relativistic corrections developed by Stevens et al. $(\mathrm{SBKJC})^{18}$ was used for the gold atoms. This ECP retains 19 explicit electrons. For geometry optimizations and Hessian calculations the basis set defined as "small" was used. This basis set consists of the Gaussian contracted SBKJC basis set $^{17}$ augmented with one set of $f$ polarization functions (exponent $=0.89$ ) and one $s$ and one set of $p$ diffuse functions (both exponents $=0.01$ ) centered on the gold atoms. The standard contracted $6-31++\mathrm{G}^{* *}$ (Ref. 19) basis set was centered on each hydrogen atom. A "large" basis set was used for single point calculations on geometries optimized with the small basis set. The large basis set consists of the completely uncontracted SBKJC basis set augmented with a $3 f / 2 g$ set of polarization functions ( $f$ function exponents $=2.00,0.84,0.31 ; \mathrm{g}$ function exponents $=1.90,0.69)$ and one $s$ and one set of $p$ diffuse functions (both exponents $=0.01$ ). The exponents of the polarization functions were optimized using MP2 calculations for atomic Au. The standard aug-cc-pVTZ ${ }^{20}$ basis set was centered on each hydrogen atom. In all calculations spherical harmonic basis functions were used with five $d$ orbitals and seven $f$ orbitals.

For plane wave DFT calculations we used the PerdewWang (PW91) ${ }^{21}$ functional, and the revised Perdew-BurkeErnzerhof (rPBE) exchange-correlation functional. ${ }^{22}$ The projector-augmented wave function pseudopotentials, ${ }^{23}$ as implemented in the VASP program ${ }^{24}$ was used. These
TABLE I. Bond distances $R(\AA)$, dissociation energies $D_{e}(\mathrm{eV})$ and vibration frequencies $\omega_{e}(1 / \mathrm{cm})$ of the $\mathrm{Au}_{2}$ at different level of theory.

\begin{tabular}{lccccccc}
\hline \hline & \multicolumn{3}{c}{ Small basis set } & & \multicolumn{3}{c}{ Large basis set } \\
\cline { 2 - 4 } \cline { 7 - 8 } & $\mathrm{R}(\AA)$ & $\mathrm{D}_{\mathrm{e}}(\mathrm{eV})$ & $\omega_{e}(1 / \mathrm{cm})$ & & $R(\AA)$ & $D_{e}(\mathrm{eV})$ & $\omega_{e}(1 / \mathrm{cm})$ \\
\hline $\mathrm{HF}$ & 2.615 & 0.80 & 156 & & 2.598 & 0.88 & 156 \\
$\mathrm{MP} 2$ & 2.494 & 2.24 & 190 & & 2.448 & 2.47 & 200 \\
CCSD(T) & 2.535 & 2.04 & 175 & & 2.504 & 2.14 & \\
B3LYP & 2.562 & 1.98 & 167 & & 2.539 & 2.03 & 168 \\
PW-PBE $^{\mathrm{a}}$ & & & & & 2.54 & 2.22 & \\
PW-PW91 $^{\mathrm{b}}$ & & & & & 2.528 & 2.27 & \\
cp-CCSD(T) $^{\mathrm{c}}$ & & & & & 2.488 & 2.19 & 187 \\
Experiment $^{\mathrm{d}}$ & & & & & 2.472 & 2.29 & 191 \\
\hline \hline
\end{tabular}

${ }^{\mathrm{a} D F T}$ with plane-wave basis set and PBE functional (Ref. 32).

${ }^{b}$ DFT with plane-wave basis set and PW91 functional (Ref. 31).

${ }^{\mathrm{c}}$ Counterpose-corrected $\mathrm{CCSD}(\mathrm{T})$ with all electron PJHN-4f2g1h1i basis set (Ref. 29).

${ }^{\mathrm{d}}$ Experimental values (Ref. 28).

pseudopotentials retain 11 explicit electrons for gold atom. The plane wave cutoff energy was $250 \mathrm{eV}$. The fictitious effect of the dipole and monopole (for anionic systems) moments of periodic images were removed by the method of Makov and Payne. ${ }^{25}$

The GAMESS ${ }^{26}$ suite of programs was used for all calculations except for $\operatorname{CCSD}(\mathrm{T})$ and plane wave density functional calculations which were done using MOLPRO ${ }^{27}$ and VASP ${ }^{24}$ respectively.

\section{RESULTS AND DISCUSSION}

Results for $\mathrm{Au}_{2}$ : In order to estimate the reliability of the methods and basis sets used in this paper, test calculations were carried out on $\mathrm{Au}_{2}$. The values of the bond length, dissociation energy and vibrational frequency, calculated with different methods and basis sets, are presented in Table I. The HF method is clearly unable to correctly predict the dissociation energy of the gold dimer. The predicted HF dissociation energy is only $\sim$ one-third of the experimental value. The bond distance and vibrational frequency deviate significantly from the experimental values as well. These poor results are essentially unaffected by basis set. The B3LYP results are in significantly better agreement with experiment. As for the HF results, the B3LYP predictions are $\sim$ independent of the basis set size. However, the B3LYP dissociation energy is still smaller than the experimental value by $0.28 \mathrm{eV}$. The bond distance is too large and the vibrational frequency is too small.

As one might suspect, the MP2 and $\operatorname{CCSD}(\mathrm{T})$ values are much more sensitive to the basis set. The methods that include electron correlation require large basis sets, since electrons, trying to avoid each other, need more degrees of freedom in Hilbert space. The MP2 values obtained with the small basis set are in better agreement with experiment than those obtained using the large basis set. This is an example of the cancellation of basis set error with the error introduced due to the incomplete treatment of electron correlation. However, the MP2 values are much closer to the experimental ones than are the values obtained using B3LYP. Even using the small basis set, the $\operatorname{CCSD}(\mathrm{T})$ results are in very good 
TABLE II. Small (large) basis $\mathrm{Au}_{2}^{-}$and $\mathrm{Au}_{2}$ binding energies (eV) for the first, $E_{b 1}$, and the second, $E_{b 2}, \mathrm{H}_{2}$ molecule.

\begin{tabular}{lccccc}
\hline \hline & MP2 & CCSD(T) & B3LYP & PW-PW91 & PW-rPBE \\
\hline $\mathrm{Au}_{2}, E_{b 1}$ & $0.39(0.69)$ & $0.27(0.55)$ & $0.30(0.42)$ & 0.59 & 0.42 \\
$\mathrm{Au}_{2}, E_{b 2}$ & $0.19(0.37)$ & $0.12(0.29)$ & $0.15(0.24)$ & 0.37 & 0.19 \\
$\mathrm{Au}_{2}^{-}, E_{b 1}$ & unstable & & unstable & 0.02 & unstable \\
$\mathrm{Au}_{2}^{-}, E_{b 2}$ & unstable & & unstable & unstable & unstable \\
\hline \hline
\end{tabular}

agreement with experiment, and they improve with the large basis set. The $\operatorname{CCSD}(\mathrm{T})$ values in Table I are in good agreement with experiment ${ }^{28}$ and with the results of Hess and Kaldor calculated with the basis set counterpoise-corrected relativistic $\operatorname{CCSD}(\mathrm{T})$ method and a very large basis set. ${ }^{29}$ Plane-wave DFT (PW91 ${ }^{30}$ and PBE $^{31}$ functionals) gives reasonable results.

Binding energies of molecular hydrogen. The binding energies of the first and second hydrogen molecules to the neutral gold clusters, summarized in Tables II and III, were determined from

$$
\begin{aligned}
& E_{b 1}=E\left[\mathrm{Au}_{n}\right]+E\left[\mathrm{H}_{2}\right]-E\left[\mathrm{Au}_{n} \mathrm{H}_{2}\right], \\
& E_{b 2}=E\left[\mathrm{Au}_{n} \mathrm{H}_{2}\right]+E\left[\mathrm{H}_{2}\right]-E\left[\mathrm{Au}_{n}\left(\mathrm{H}_{2}\right)_{2}\right] .
\end{aligned}
$$

Here $n=2,3$ is the number of atoms in the specific gold cluster and $E[X]$ is the energy of the molecule $X$. The binding energies of the hydrogen molecules to the negatively charged gold clusters were determined in an analogous manner. If $\mathrm{H}_{2}$ does not bind to a gold cluster as molecular hydrogen, it can still bind as two separate hydrogen atoms, as long as the reactants have sufficient energy to break the $\mathrm{H}-\mathrm{H}$ bond.

All levels of theory predict that the first $\mathrm{H}_{2}$ binds to neutral $\mathrm{Au}_{2}$ (Table II). Using the large basis set, the MP2 $(0.69 \mathrm{eV})$ and B3LYP $(0.42 \mathrm{eV})$ binding energies bracket the $\operatorname{CCSD}(\mathrm{T})$ result of $0.55 \mathrm{eV}$. The plane wave PW-PW91 and PW-rPBE methods $(0.59 \mathrm{eV}$ and $0.42 \mathrm{eV}$, respectively) are in good agreement with $\operatorname{CCSD}(\mathrm{T})$. The second $\mathrm{H}_{2}$ binds to $\mathrm{Au}_{2} \mathrm{H}_{2}$ with a smaller bonding energy than that of the first $\mathrm{H}_{2}$ (cf. Table II). PW-PW91 predicts a very small $(0.02 \mathrm{eV})$
TABLE III. Small (large) basis $\mathrm{Au}_{3} / \mathrm{Au}_{3}^{-}$binding energies (eV) for the first, $E_{b 1}$, and the second, $E_{b 2}, \mathrm{H}_{2}$ molecule.

\begin{tabular}{lccccc}
\hline \hline & MP2 & CCSD(T) & B3LYP & PW-PW91 & PW-rPBE \\
\hline $\mathrm{Au}_{3}, E_{b 1}$ & $0.58(0.90)$ & $0.41(0.71)$ & $0.39(0.52)$ & 0.71 & 0.53 \\
$\mathrm{Au}_{3}, E_{b 2}$ & $0.17(0.34)$ & $0.08\left(0.25^{\mathrm{a}}\right)$ & $0.11(0.18)$ & 0.26 & 0.08 \\
$\mathrm{Au}_{3}^{-}, E_{b 1}$ & unstable & & unstable & 0.05 & unstable \\
$\mathrm{Au}_{3}^{-}, E_{b 2}$ & unstable & & unstable & unstable & unstable \\
\hline
\end{tabular}

${ }^{\mathrm{a}} \mathrm{CCSD}(\mathrm{T}) /$ large values was estimated based on $\operatorname{CCSD}(\mathrm{T}) / \mathrm{small}, \mathrm{MP} 2 / \mathrm{small}$ and MP2/large values (see explanation in the text).

binding energy for the first $\mathrm{H}_{2}+\mathrm{Au}_{2}^{-}$. All other methods find that molecular hydrogen does not bind to the negatively charged $\mathrm{Au}_{2}^{-}$cluster.

To estimate the error introduced by using the small basis set for geometry optimizations, the geometries of $\mathrm{Au}_{2} \mathrm{H}_{2}$ were reoptimized using the large basis set. The difference in large basis set binding energies obtained using the small vs large basis sets geometries is only $0.02 \mathrm{eV}$. Geometry optimizations for all other complexes were therefore carried out using the small basis set. However, note that although the basis set effects on predicted geometries are small, there are significant basis set effects on the predicted binding energies. For all methods, the binding energies increase when the large basis set is employed.

The equilibrium geometries of the gold clusters with adsorbed hydrogen molecules are shown in Fig. 1. All complexes are planar. The two $\mathrm{H}_{2}$ hydrogen atoms coordinate to the same gold atom, in a symmetric $\left(C_{2 v}\right)$ manner. The lengths of the $\mathrm{H}-\mathrm{H}$ bonds increase by $0.05-0.10 \AA$, compared with free hydrogen molecule $(0.734 \AA)$. Addition of the first $\mathrm{H}_{2}$ stretches the $\mathrm{Au}-\mathrm{Au}$ bond length by $\sim 0.04 \AA$. The effect of the second $\mathrm{H}_{2}$ is much smaller, consistent with the smaller binding energy.

The binding energies of the first and second hydrogen molecules to the $\mathrm{Au}_{3}$ clusters are given in Table III. Again, the negatively charged cluster does not bind molecular hydrogen. The binding energy of the first $\mathrm{H}_{2}$ to the neutral $\mathrm{Au}_{3}$ cluster according to $\operatorname{CCSD}(\mathrm{T}) /$ large is $0.71 \mathrm{eV}$. It was not possible to carry out the $\operatorname{CSSD}(\mathrm{T})$ calculation of the binding energy of the second $\mathrm{H}_{2}$ to the $\mathrm{Au}_{3}$ cluster with the large

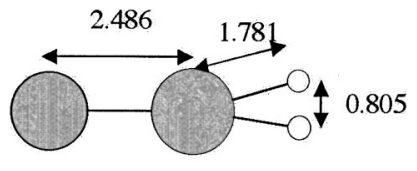

$\mathrm{Au}_{2} \mathrm{H}_{2}{ }^{1} \mathrm{~A}_{1}$

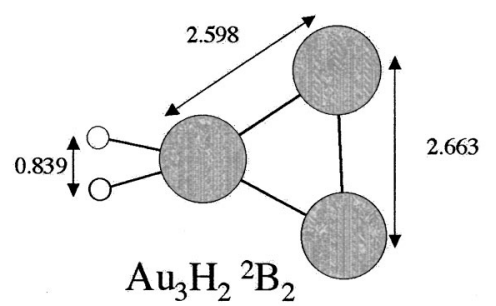

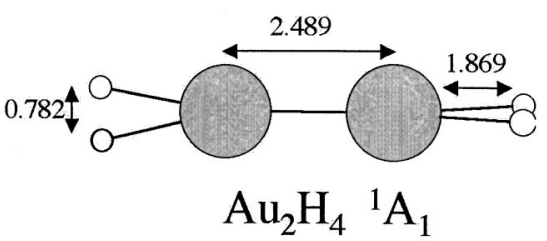

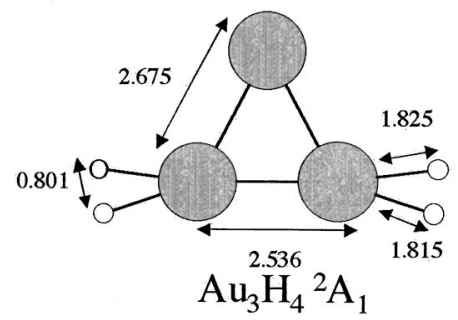

FIG. 1. MP2/large optimized geometries for lowest energy ground states of $\mathrm{Au}_{2} \mathrm{H}_{2}, \mathrm{Au}_{2} \mathrm{H}_{4}, \mathrm{Au}_{2} \mathrm{H}_{2}^{-}$, and $\mathrm{Au}_{2} \mathrm{H}_{4}^{-}$complexes. Bond distances are in angstroms. 


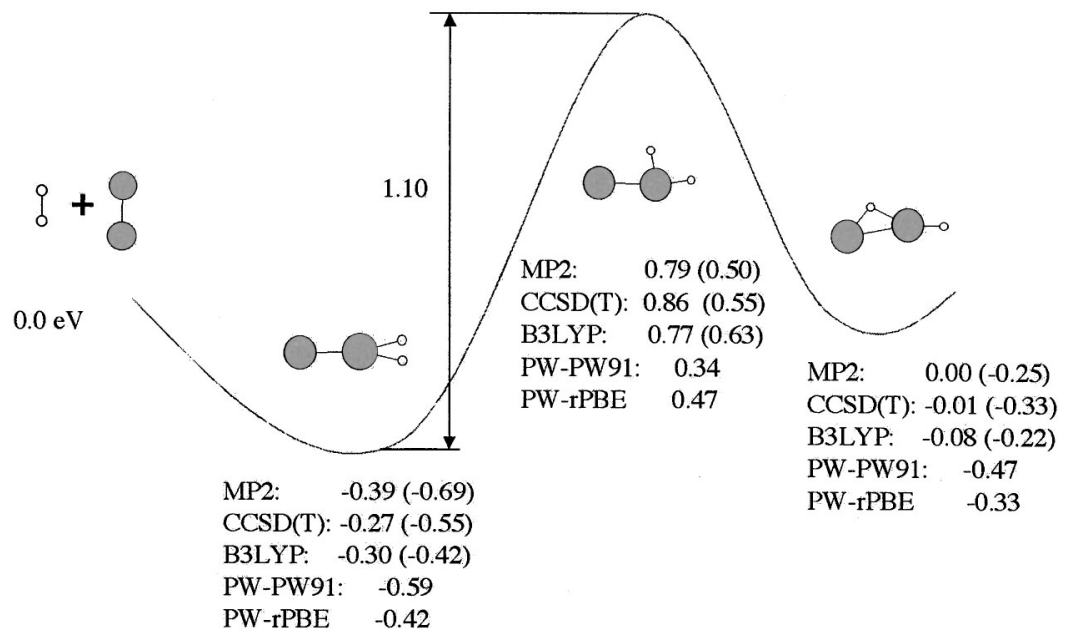

FIG. 2. Reaction path for $\mathrm{H}_{2}$ dissociation on neutral $\mathrm{Au}_{2}$, with relative energies of minima and transition state. The energies calculated with the large basis set are in parentheses. basis set because of the large number (nearly 400) of basis functions. However, the $\operatorname{CCSD}(\mathrm{T}) /$ large results can be estimated from the CCSD(T)/small calculations and MP2 calculations with both basis sets, in analogy with the procedures used in the GN methods. ${ }^{32}$ Assuming that $E[\mathrm{CCSD}(\mathrm{T}) /$ large $]-E[\mathrm{CCSD}(\mathrm{T}) /$ small $]$ is the same as the corresponding MP2 energy difference, the relative $\operatorname{CCSD}(\mathrm{T})$ / large energy at the highest transition state is estimated to be $0.08 \mathrm{eV}+0.17 \mathrm{eV}=0.25 \mathrm{eV}$. The MP2 and B3LYP and PWrPBE binding energies are in good and PW-PW91 values are in perfect agreement with the $\operatorname{CCSD}(\mathrm{T})$ values.

Dissociation of molecular hydrogen on gold clusters. The reaction path for dissociation of molecular hydrogen on the neutral $\mathrm{Au}_{2}$ cluster is presented in Fig. 2. The energies of the transition state and reaction products are shown relative to the energy of the separated $\mathrm{Au}_{2}$ and $\mathrm{H}_{2}$. The values obtained from calculations using the large basis set are shown in parentheses. The relative energies of the transition state and reaction products obtained with most methods are in good agreement with each other. However, the PW-PW91 calculations underestimate the barrier by $0.21 \mathrm{eV}$ and overestimate the stability of the product by $0.14 \mathrm{eV}$. Still, this should be considered reasonably accurate if the calculations are used for answering qualitative questions. The PW-rPBE method gives much better results than PW-PW91 for the barrier and stability of the product. The PW-rPBE energy of the product is in perfect agreement with $\operatorname{CCSD}(\mathrm{T})$ and the barrier is underestimated by only $0.08 \mathrm{eV}$. Of course, this method has been parametrized for systems like the one under study; it is not clear how well the success seen here will carry over to other systems. The $\operatorname{CCSD}(\mathrm{T})$ activation energy for the dissociation of molecular hydrogen on the $\mathrm{Au}_{2}$ cluster is $1.10 \mathrm{eV}$ (0.55 eV above separated reactants). The $\mathrm{Au}_{2}$ cluster with one bridging and one terminal hydrogen atom is $0.22 \mathrm{eV}$ higher in energy than $\mathrm{Au}_{2}$ with an adsorbed $\mathrm{H}_{2}$ molecule, so the dissociation is predicted to be mildly endothermic.

All methods make the same qualitative predictions: $\mathrm{H}_{2}$ binds to $\mathrm{Au}_{2}$ and has a fairly high barrier to dissociation. The state with the dissociated hydrogen is metastable with respect to the adsorbed molecular hydrogen. The barriers are such that the adsorbed $\mathrm{H}_{2}$ would much rather desorb than dissociate.

As noted above, while there is no stable $\mathrm{Au}_{2}^{-} \cdot \mathrm{H}_{2}$ cluster, there is a stable $\mathrm{Au}_{2} \mathrm{H}_{2}^{-}$species in which the molecular hydrogen has dissociated. This is illustrated in Fig. 3. $\operatorname{CCSD}(\mathrm{T}) /$ large predicts the largest barrier for this reaction $\left(0.93 \mathrm{eV}\right.$ relative to separated $\mathrm{Au}_{2}^{-}$and $\left.\mathrm{H}_{2}\right)$. The transition state has an asymmetrically bound $\mathrm{H}_{2}$ molecule with a sig-

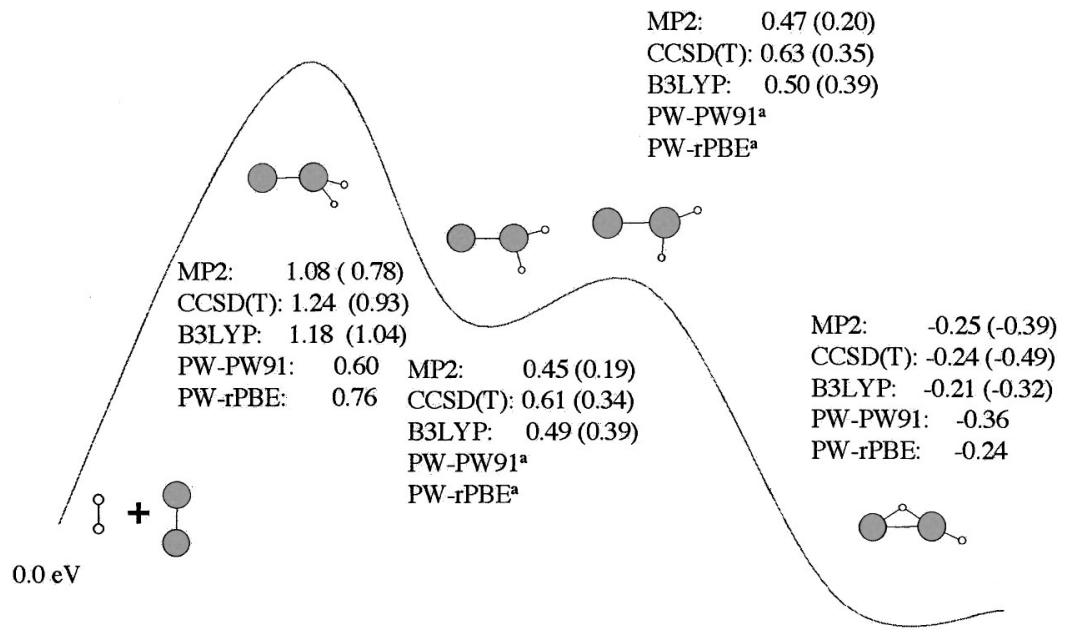

FIG. 3. Reaction path for $\mathrm{H}_{2}$ dissociation on negatively charged $\mathrm{Au}_{2}^{-}$, with relative energies of minima and transition states. The energies calculated with the large basis set are in parentheses. There is a shoulder instead of a minimum and transition state on the PW-PW91 and PW-rPBE reaction paths. 


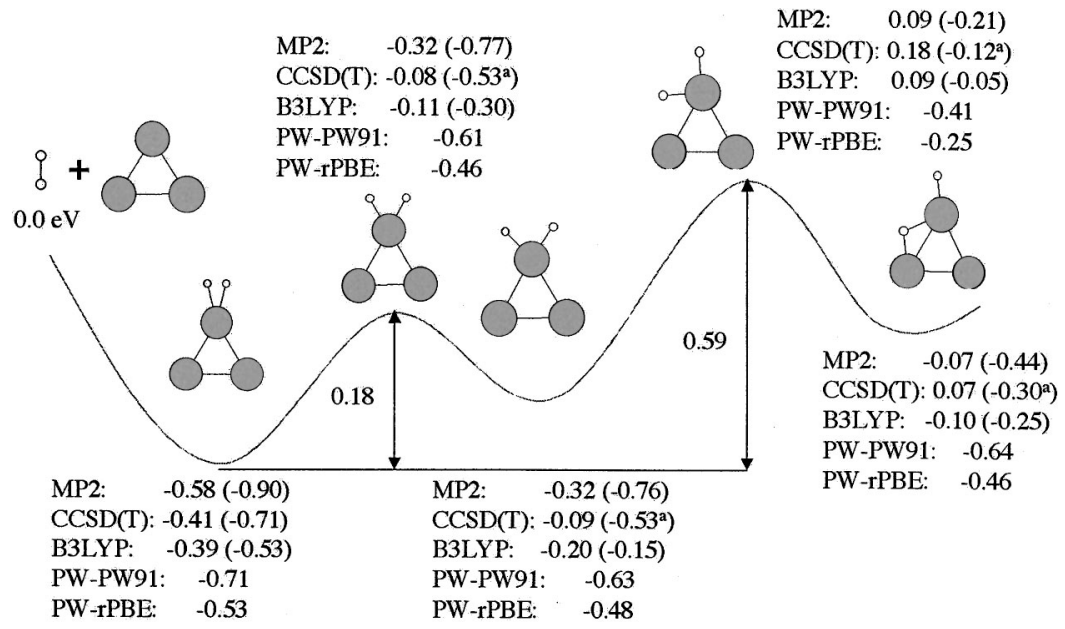

FIG. 4. Reaction path for $\mathrm{H}_{2}$ dissociation on neutral $\mathrm{Au}_{3}$, with relative energies of minima and transition states. The energies calculated with the large basis set are in parentheses. CCSD(T)/large values were estimated based on CCSD(T)/small, MP2/small, and MP2/ large values (see explanation in the text). nificantly stretched $(1.085 \AA) \mathrm{H}-\mathrm{H}$ bond, compared with free hydrogen molecule $(0.734 \AA)$. There is one very shallow minimum and one very low energy transition state on this reaction path. The transition state is only $0.01 \mathrm{eV}$ higher in energy than the minimum and therefore may not exist. The presence of these stationary points illustrates that even such a seemingly simple reaction can have a nontrivial potential energy surface. However, these local minima and transition state probably do not play a significant role in the mechanism of $\mathrm{H}_{2}$ dissociation. The PW-PW91 and PW-rPBE DFT methods predict the existence of a shoulder on this reaction path instead of a very shallow minimum and transition state with very small activation energy. Interestingly, for this reaction PW-rPBE gives a barrier that is in better agreement with the $\operatorname{CCSD}(\mathrm{T})$ result than the PW-PW91 barrier. However, the PW-PW91 energy of the product relative to reactants is closer to the $\operatorname{CCSD}(\mathrm{T})$ value than is the PW-rPBE product energy. Both functionals underestimate the barrier and stability of the product.

The dissociation of $\mathrm{H}_{2}$ on the $\mathrm{Au}_{3}$ cluster (Fig. 4) is more complex and characterized by several minima and transition states. Because of the large number of basis functions and the low symmetry of some of the stationary points, it was not possible to carry out open-shell $\operatorname{CCSD}(\mathrm{T})$ calculations with the large basis set for all of these species. However, the CCSD(T)/large energies of $\mathrm{Au}_{3} \mathrm{H}_{2}$ can be estimated using the same procedure as was used to estimate the binding energy of the second $\mathrm{H}_{2}$ to the $\mathrm{Au}_{3}$ cluster. The dependence of the results on the size of the basis set is quite significant for this reaction. All methods except PW-PW91 are in good agreement with each other. The PW-PW91 and PW-rPBE potential energy surfaces appear to be too flat.

After bonding to the $\mathrm{Au}_{3}$ cluster, with a binding energy of $0.71 \mathrm{eV}, \mathrm{H}_{2}$ can dissociate through a symmetric transition state with an estimated $\operatorname{CCSD}(\mathrm{T})$ activation energy of 0.18 $\mathrm{eV}$. However, the minimum on the potential energy surface that corresponds to two hydrogen atoms, symmetrically attached to one gold atom, is almost isoenergetic with this transition state. Therefore, the lifetime of $\mathrm{a} \mathrm{Au}_{3}$ cluster with two hydrogen atoms attached to one gold atom is likely to be very small. In order to form the $\mathrm{Au}_{3} \mathrm{H}_{2}$ complex with dissociated hydrogen and a significant lifetime, the reaction must proceed further through an asymmetric transition state towards the complex with one bridging and one terminating hydrogen atom. The estimated MP2 and $\operatorname{CCSD}(\mathrm{T})$ activation energies for this reaction, with respect to the $\mathrm{Au}_{3} \mathrm{H}_{2}$ complex with molecular hydrogen, are 0.69 and $0.59 \mathrm{eV}$, respectively. It is important to point out that these values do not depend on the basis set used. For comparison PW-PW91 and PW-rPBE values are $0.30 \mathrm{eV}$ and $0.28 \mathrm{eV}$, respectively.

The mechanism of $\mathrm{H}_{2}$ dissociation on the negatively charged $\mathrm{Au}_{3}^{-}$cluster is shown in Fig. 5. Molecular hydrogen does not form a stable complex with this gold anion. The $\operatorname{CCSD}(\mathrm{T}) /$ large barrier for the $\mathrm{H}_{2}$ dissociation is $1.39 \mathrm{eV}$. B3LYP, MP2, and CCSD $(\mathrm{T})$ give very similar values for the barrier, whereas the PW-PW91 and PW-rPBE values are again too low.

Finally, in order to explore the possibility of other bridging structures on the small gold clusters, MP2 calculations were performed to search for double-bridged structures that might correspond to minima on the potential energy surfaces of $\mathrm{H}_{2}$ with neutral and negatively charged $\mathrm{Au}_{2}$ and $\mathrm{Au}_{3}$ clusters. No such local minima have been found. Similarly, for $\mathrm{Au}_{3}$ and $\mathrm{Au}_{3}^{-}$no stable complexes with $\mathrm{H}$ atom coordinated to all three gold atoms were found.

\section{SUMMARY}

It has been shown that one and even two $\mathrm{H}_{2}$ molecules easily bind to neutral $\mathrm{Au}_{2}$ and $\mathrm{Au}_{3}$ clusters. According to the best $\operatorname{CCSD}(\mathrm{T})$ calculations, the activation energies for dissociation of $\mathrm{H}_{2}$ adsorbed on neutral $\mathrm{Au}_{2}$, and $\mathrm{Au}_{3}$ clusters are $1.10 \mathrm{eV}$ and $0.59 \mathrm{eV}$. On the other hand, the activation energies with respect to separate $\mathrm{Au}$ clusters and $\mathrm{H}_{2}$ molecules are only $0.55 \mathrm{eV}$ and $-0.12 \mathrm{eV}$ (transition state is lower in energy than separated $\mathrm{Au}_{3}$ and $\mathrm{H}_{2}$ ). This means that some $\mathrm{H}_{2}$ molecules can dissociate on these clusters directly without formation of weakly bound $\mathrm{H}_{2}$-gold cluster complexes. But since these weak complexes correspond to global minima on the potential energy surfaces, their population at equilibrium should be significant.

Molecular hydrogen does not form stable complexes with negatively charged $\mathrm{Au}_{2}^{-}$and $\mathrm{Au}_{3}^{-}$clusters. The dissociation barriers for $\mathrm{H}_{2}$ on these clusters (with respect to the 


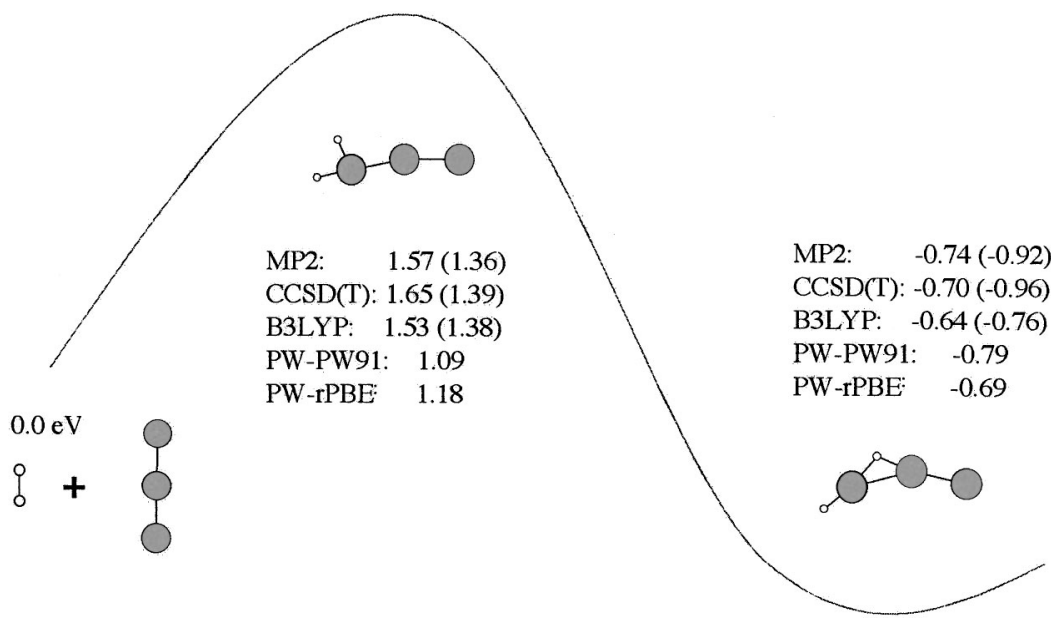

FIG. 5. Reaction path for $\mathrm{H}_{2}$ dissociation on neutral $\mathrm{Au}_{3}^{-}$, with relative energies of minima and transition states. The energies calculated with the large basis set are in parentheses. separate cluster and $\left.\mathrm{H}_{2}\right)$ are $0.93 \mathrm{eV}\left(\mathrm{Au}_{2}^{-}\right)$and $1.39 \mathrm{eV}$ $\left(\mathrm{Au}_{3}^{-}\right)$. Since the energies of $\mathrm{Au}_{2}^{-}$and $\mathrm{Au}_{3}^{-}$with adsorbed dissociated $\mathrm{H}_{2}$ are $0.49 \mathrm{eV}$ and $0.96 \mathrm{eV}$, respectively, lower than the energies of separated $\mathrm{H}_{2}$ and gold clusters, formation of these adsorbed complexes is possible if the reactants have sufficient energy to overcome the barriers and if collisions with an inert gas dissipate the adsorption energy.

It is interesting to compare the adsorption characteristics of $\mathrm{H}_{2}$ vs $\mathrm{O}_{2}$ to small gold clusters. In contrast to oxygen adsorption $^{7}$ the DFT methods perform reasonably well for predicting geometries and hydrogen adsorption on small gold clusters, at least with regard to the prediction of binding energies. The plane wave basis set with the PW91 and rPBE functionals, predicts binding energies that are very similar to the $\operatorname{CCSD}(\mathrm{T})$ values with the large Gaussian basis set. However, the PW-PW91 and PW-rPBE methods tend to underestimate activation energies.

The small basis set used here appears to be sufficient for optimization of the geometries and calculations of the relative energies of minima and transition states. For quantitative prediction of binding energies of $\mathrm{H}_{2}$ to gold clusters it is necessary to use the large basis set, as there are significant basis set effects. The small basis set appears to be sufficient for predicting the values of $\mathrm{O}_{2}$ binding energies. ${ }^{33}$ The difference may be associated with the small number of Gaussian functions on hydrogen atom in the 6-31G(d) basis set.

The molecular hydrogen adsorption on small gold clusters is very different from that of molecular oxygen. ${ }^{7} \mathrm{H}_{2}$ coordinates to gold clusters with both hydrogen atoms, whereas, only one oxygen atom of $\mathrm{O}_{2}$ coordinates to a gold cluster. Molecular hydrogen does not bind to negatively charged $\mathrm{Au}_{n}^{-}$clusters. In contrast, oxygen binding mostly depends on the presence of unpaired electrons on the gold clusters, and is associated with partial electron transfer from the gold cluster to $\mathrm{O}_{2}$. Of the clusters studied in this paper, only neutral $\mathrm{Au}_{3}$ can bind individual $\mathrm{H}_{2}$ and $\mathrm{O}_{2}$ molecules, with $\mathrm{O}_{2}$ and $\mathrm{H}_{2}$ binding energies of $0.17 \mathrm{eV}$ and $0.71 \mathrm{eV}$, respectively. $\mathrm{H}_{2}$ binds to neutral $\mathrm{Au}_{2}$, while $\mathrm{O}_{2}$ does not. On the other hand, $\mathrm{O}_{2}$ binds to $\mathrm{Au}_{2}^{-}$, while $\mathrm{H}_{2}$ does not. Therefore, one can speculate that simultaneous adsorptions of $\mathrm{O}_{2}$ and $\mathrm{H}_{2}$ are possible only on neutral clusters with an odd number of gold atoms. Exploring the validity of this hypoth- esis and its relevance to catalytic properties of small gold clusters will be the focus of future work.

\section{ACKNOWLEDGMENTS}

The authors thank Dr. Michael Schmidt and Dr. Dmitri Fedorov for helpful discussions. This work was supported by AFOSR through a DURINT grant. The calculations in this work were performed in part on an IBM workstation cluster made possible by grants from IBM in the form of a Shared University Research grant and the United States Department of Energy.

${ }^{1}$ R. J. Bartlett, J. Phys. Chem. 93, 1697 (1989); C. Hampel, K. Peterson, and H.-J. Werner, Chem. Phys. Lett. 190, 1 (1992), and references therein. The program to compute the perturbative triples corrections has been developed by M. J. O. Deegan and P. J. Knowles (1992).

${ }^{2}$ A. Sarkany and Z. Revay, Appl. Catal., A 243, 347 (2003).

${ }^{3}$ C. Mohr, H. Hofmeister, and P. Claus, J. Catal. 213, 86 (2003); C. Mohr, H. Hofmeister, J. Radnik, and P. Claus, J. Am. Chem. Soc. 125, 1905 (2003).

${ }^{4}$ J. Jia, K. Haraki, J. N. Kondo, K. Domen, and K. Tamaru, J. Phys. Chem. B 104, 11153 (2000); A. Sarkany and Z. Revay, Appl. Catal., A 243, 347 (2003); T. V. Choudhary, C. Sivadinarayana, A. K. Datye, D. Kumar, and D. W. Goodman, Catal. Lett. 86, 1 (2003).

${ }^{5}$ G. C. Bond and D. T. Thompson, Catal. Rev. - Sci. Eng. 41, 319 (1999); G. J. Hutchins, Catal. Today 72, 11 (2002); G. C. Boyd, ibid. 72, 5 (2002); M. Haruta, ibid. 36, 153 (1997); H. Haruta and M. Date, Appl. Catal., A 222, 427 (2001).

${ }^{6}$ B. Hammer and J. K. Norskov, Adv. Catal. 45, 71 (2000); P. Kratzer and M. Scheffler, Comput. Sci. Eng. 3, 16 (2001); J. K. Norskov, "Reaction kinetics and the development of catalytic processes," 1999, pp. 3-10; Nature (London) 414, 405 (2001).

${ }^{7}$ S. A. Varganov, R. M. Olson, M. S. Gordon, and H. Metiu, J. Chem. Phys. 119, 2531 (2003).

${ }^{8}$ E. Wahlstrom, N. Lopez, R. Schaub, P. Thostrup, A. Ronnau, C. Africh, E. Laegsgaard, J. K. Norskov, and F. Besenbacher, Phys. Rev. Lett. 90, 026101 (2003); R. Schaub, E. Wahlstrom, A. Ronnau, E. Laegsgaard, I. Stensgaard, and F. Besenbacher, Science 299, 377 (2003).

${ }^{9}$ A. Vijay, G. Mills, and H. Metiu, J. Chem. Phys. 118, 6536 (2003); U. Diebold, Surf. Sci. Rep. 48, 53 (2003).

${ }^{10}$ R. Schaub, R. Thostrup, N. Lopez, E. Laegsgaard, I. Stensgaard, J. K. Norskov, and F. Besenbacher, Phys. Rev. Lett. 87, 266104 (2001); U. Diebold, Surf. Sci. Rep. 48, 53 (2003).

${ }^{11}$ A. Sanchez, S. Abbet, U. Heiz, W. D. Schneider, H. Hakkinen, R. N. Barnett, and U. Landman, J. Phys. Chem. A 103, 9573 (1999).

${ }^{12}$ C. Møller and M. S. Plesset, Phys. Rev. 46, 618 (1934).

${ }^{13}$ K. Ishida, K. Morokuma, and A. Komornicki, J. Chem. Phys. 66, 2153 (1977).

${ }^{14}$ C. Gonzales and H. B. Schlegel, J. Chem. Phys. 90, 2154 (1989). 
${ }^{15}$ T. J. Lee and D. Jayatilaka, Chem. Phys. Lett. 201, 1 (1993); T. J. Lee, A. P. Rendell, K. G. Dyall, and D. Jayatilaka, J. Chem. Phys. 100, 7400 (1994).

${ }^{16}$ P. J. Knowles, C. Hampel, and H.-J. Werner, J. Chem. Phys. 99, 5219 (1993).

${ }^{17}$ A. D. Becke, J. Chem. Phys. 98, 5648 (1993); P. J. Stephens, F. J. Devlin, C. F. Chablowski, and M. J. Frisch, J. Phys. Chem. 98, 11623 (1994); R. H. Hertwig and W. Koch, Chem. Phys. Lett. 268, 345 (1997).

${ }^{18}$ W. J. Stevens, M. Krauss, H. Basch, and P. G. Jasien, Can. J. Chem. 70, 612 (1992)

${ }^{19}$ W. J. Hehre, R. Ditchfield, and J. A. Pople, J. Chem. Phys. 56, 2257 (1972).

${ }^{20}$ T. H. Dunning, Jr., J. Chem. Phys. 90, 1007 (1989). Basis sets were obtained from the Extensible Computational Chemistry Environment Basis Set Database, Version 6/19/03, as developed and distributed by the Molecular Science Computing Facility, Environmental and Molecular Sciences Laboratory which is part of the Pacific Northwest Laboratory, P.O. Box 999, Richland, Washington 99352, USA, and funded by the U.S. Department of Energy. The Pacific Northwest Laboratory is a multiprogram laboratory operated by Battelle Memorial Institute for the U.S. Department of Energy under Contract No. DE-AC06-76RLO 1830.

${ }^{21}$ Y. Wang and J. P. Perdew, Phys. Rev. B 44, 13298 (1991).
${ }^{22}$ B. Hammer, L. B. Hansen, and J. K. Norskov, Phys. Rev. B 59, 7413 (1999).

${ }^{23}$ G. Kresse and J. Joubert, Phys. Rev. B 59, 1758 (1999).

${ }^{24}$ G. Kresse and J. Hafner, Phys. Rev. B 47, 558 (1993); 49, 14251 (1994); G. Kresse and J. Furthmuller, Comput. Mater. Sci. 6, 15 (1996); Phys. Rev. B 54, 11169 (1996).

${ }^{25}$ G. Makov and M. C. Payne, Phys. Rev. B 51, 4014 (1995).

${ }^{26}$ M. W. Schmidt, K. K. Baldridge, J. A. Boatz et al., J. Comput. Chem. 14, 1347 (1993); G. D. Fletcher, M. W. Schmidt, and M. S. Gordon, Adv. Chem. Phys. 110, 267 (1999); G. D. Fletcher, M. W. Schmidt, B. M. Bode, and M. S. Gordon, Comput. Phys. Commun. 128, 190 (2000).

${ }^{27}$ MOLPRO is a package of $a b$ initio programs written by H.-J. Werner and P. J. Knowles, with contributions from R. D. Amos, A. Bernhardsson, A. Berning et al.

${ }^{28}$ K. P. Huber and G. Herzberg, Molecular Spectra and Molecular Structure. IV. Constants of Diatomic Molecules (Van Nostrand, New York, 1979).

${ }^{29}$ B. A. Hess and U. Kaldor, J. Chem. Phys. 112, 1809 (2000).

${ }^{30}$ G. Mills, M. S. Gordon, and H. Metiu, Chem. Phys. Lett. 359, 493 (2002).

${ }^{31}$ H. Hakkinen and U. Landman, J. Am. Chem. Soc. 123, 9704 (2001).

${ }^{32}$ L. A. Curtiss, K. Raghavachari, P. C. Redfern, V. Rassolov, and J. A. Pople, J. Chem. Phys. 109, 7764 (1998).

${ }^{33}$ S. A. Varganov, R. M. Olson, M. S. Gordon, and H. Metiu (unpublished). 